\title{
Designing Backstepping Control System for Hypersonic Vehicle Based on Feedback Linearization
}

\author{
Jianli Wei ${ }^{1}$ and Huan Chen ${ }^{2}$ \\ ${ }^{1}$ College of Astronautics, Northwestern Polytechnical University, 127 West Youyi Road, Xian, Shaanxi 710072, China \\ ${ }^{2}$ Beijing Aerospace Jiacheng Precision S\&T Co., Ltd., Beijing 102600, China
}

Correspondence should be addressed to Jianli Wei; weijianli@mail.nwpu.edu.cn

Received 11 April 2015; Revised 24 July 2015; Accepted 30 July 2015

Academic Editor: Paul Williams

Copyright (C) $2015 \mathrm{~J}$. Wei and H. Chen. This is an open access article distributed under the Creative Commons Attribution License, which permits unrestricted use, distribution, and reproduction in any medium, provided the original work is properly cited.

A hypersonic vehicle uses the airbreathing scramjet engine and the airframe and engine integrated design. Therefore, there is a strong cross-coupling effect among its aerodynamic force, thrust, structure, and control. The nonlinearity and uncertainty of the model cause difficulties in control system design. Considering the nonlinearity, coupling characteristics, and aerodynamic parametric uncertainty of its longitudinal dynamic model, we design the control law for its altitude system and velocity system based on the adaptive backstepping control method. Because of the feedback linearization method, we introduce the constraints of the flight vehicle's actuator into the design, obtaining the robust adaptive control system constrained by the actuator of the flight vehicle. To avoid the high-order derivation problem of the feedback linearization method and the derivation of the virtual control volume in adaptive backstepping control method, we use the arbitrary-order robust exact differentiator to solve the high-order derivatives in feedback linearization and utilize the command filter to obtain the virtual control volume and its derivatives. The simulation results show that the robust adaptive control system we designed can achieve the error-free tracking of altitude and velocity command. It can well overcome the influence of structural parameters, aerodynamic parametric uncertainty, and disturbances; meanwhile, the control command can satisfy the constraints of the actuator.

\section{Introduction}

A hypersonic vehicle flies fast and has a large altitude range, and its parameters vary with time greatly. When its flight attitude changes quickly, it is a highly coupled nonlinear time varying system. The traditional small disturbance linearization theory and the triple-path independent (comprehensive) control system design method based on the coefficient-freeze hypothesis are not pertinent. During the control system design, the coupling effect must be taken into account, and a complex system should be decoupled [1]

The feedback linearization method, which is developed based on the differential geometrical theory, can achieve the precise linearization of the input and output of a nonlinear mathematical model through appropriate nonlinear state transformation and feedback transformation. It can not only effectively avoid neglecting errors caused by the high-order terms of a nonlinear model but also transform the complicated nonlinear problem of a hypersonic vehicle into a linear problem, thus achieving the decoupling of altitude and velocity. Wang and Stengel [2] designed a robust flight control system using integrated nonlinear dynamic inversion and estimated the robustness of the system using Mont Carlo method. Atassi and Khalil [3] studied the application of the feedback linearization method to different control systems and verified that it can achieve effective decoupling and coordinated control. Sigthorsson et al. [4, 5] studied a robust linear output feedback control and sensor placement for an airbreathing hypersonic vehicle. Taking into account the input coupling and modeling uncertainty problems that exist in the application of the feedback linearization method, Rehman et al. $[6,7]$ proposed the robust feedback linearization and minimax linear quadratic regulator (LQR) hybrid control method. Based on the feedback linearization, Sun et al. [8] designed an adaptive integral-sliding-mode fault-tolerant controller using robust fault-tolerant tracking 
control method; it can effectively suppress external interferences and actuator failures.

Considering the strong coupling nonlinear characteristics of a hypersonic vehicle and its feedback linearization, we propose the backstepping control method, which is a very effective nonlinear robust control method. The backstepping control method is an adaptive controller design method proposed by Kokotoivc and his collaborators for solving nonlinear system control problems [9]. The design of a backstepping controller is a recursive process. It starts from the last step and from rear to front, repeatedly selects the appropriate Lyapunov function, recurses step by step to construct the auxiliary control input, and separates the design of a whole system into one of a series of low-order subsystems, eventually obtaining a stable control algorithm. The backstepping control method can guarantee the closed-loop stability and tracking progress of the hypersonic vehicle [10]. According to the Euler approximate and discrete model of a hypersonic vehicle, Gao et al. [11, 12] proposed the discrete adaptive control method based on the backstepping control method and carried out simulations to verify that the discrete adaptive control method can guarantee the agreement and final value boundedness between the tracking errors and fuzzy adaptive parametric errors. Jianfei et al. [13] proposed the adaptive backstepping terminal sliding mode control method for an uncertain nonlinear system with a class of strict feedback parameters, which enabled the last state of the hypersonic vehicle to converge within a limited time; the hypersonic vehicle was thus robust to both matching and nonmatching uncertainties. Xu et al. [14-17] designed a discrete adaptive neural network controller based on backstepping theory for hypersonic vehicle. Using longitudinal dynamic model of hypersonic vehicle, $\mathrm{Xu}$ et al. [18] designed a MIMO adaptive sliding controller, which shows robustness for parameter uncertainty. Considering that the control system actuators and flight status of hypersonic vehicle are limited, Farrell et al. [19-21] proposed a new concept; they introduced constraints of actuators into the control law, using online estimation method, and introduced constraints of amplitude, angular velocity, and bandwidth into actuators and flight status of uncertain nonlinear systems, and then an adaptive backstepping controller with constraints was designed. Tee et al. [22] proposed a new backstepping method based on Barrier Lyapunov Function; it can solve control problems of nonlinear systems including output and state constraints.

Therefore, to solve velocity and altitude tracking control problem of a hypersonic vehicle, we combine the feedback linearization method with the backstepping control method and introduce the actuator constraints into the control law design. This method can decouple the altitude and velocity of the hypersonic vehicle and enhance the control system's robustness to parametric uncertainty and its commandtracking speed, within a reasonable control command scope.

\section{Longitudinal Model of Hypersonic Vehicle}

We study the airbreathing generic hypersonic vehicle developed by the Multidisciplinary Flight Dynamics and Control
Laboratory of California State University at Los Angeles (CSULA-GHV) $[23,24]$; its longitudinal nonlinear dynamics model is as follows:

$$
\begin{aligned}
\dot{V} & =\frac{(T \cos \alpha-D)}{m}-g \sin \theta, \\
\dot{H} & =V \sin \theta \\
\dot{\theta} & =\frac{(T \sin \alpha+L)}{m V}-\frac{g \cos \theta}{V}, \\
\dot{\omega}_{z} & =\frac{M_{z}}{J_{z}} \\
\dot{\alpha} & =\omega_{z}-\dot{\theta} .
\end{aligned}
$$

Aerodynamic parameters are shown as follows:

$$
\begin{aligned}
C_{L}= & 0.04264+0.98065 \alpha+0.26716 \delta_{T}-0.06332 \delta_{z} \\
& -0.00922 M+0.45887 \alpha \delta_{T}+0.15780 \alpha \delta_{z} \\
& -0.04411 \alpha M-0.01856 \delta_{T} M+0.00136 \delta_{z} M \\
& +0.16144 \alpha^{2}+0.00049 M^{2}, \\
C_{D}= & 0.00099+0.01967 \alpha-0.04902 \delta_{T}+0.01098 \delta_{z} \\
& +0.00072 M-0.02242 \alpha \delta_{T}-0.15628 \alpha \delta_{z} \\
& -0.00059 \alpha M+0.00343 \delta_{T} M-0.00035 \delta_{z} M \\
& +0.71317 \alpha^{2}-5.92 \times 10^{-5} \times M^{2}, \\
C_{T}= & 0.01405+0.04084 \alpha+0.09316 \delta_{T}+0.00046 \delta_{z} \\
& -0.00304 M+0.17655 \alpha \delta_{T}-0.00048 \alpha \delta_{z} \\
& -0.00491 \alpha M-0.00623 \delta_{T} M-5.27 \times 10^{-5} \\
& \times \delta_{z} M-0.08687 \alpha^{2}+0.00015 M^{2}, \\
C_{m}= & -0.01370-0.19045 \alpha-0.13389 \delta_{T}+0.04217 \delta_{z} \\
& +0.00415 M-0.22796 \alpha \delta_{T}-0.10083 \alpha \delta_{z} \\
& +0.01083 \alpha M+0.00948 \delta_{T} M-0.00094 \delta_{z} M \\
& 0.11234 \alpha^{2}-0.00023 M^{2} .
\end{aligned}
$$

The model of an airbreathing engine can be equalized into a second-order model:

$$
\ddot{\delta}_{T}=-2 \xi_{n} \omega_{n} \dot{\delta}_{T}-\omega_{n}^{2} \delta_{T}+\omega_{n}^{2} \delta_{T c},
$$

where $\xi_{n}$ and $\omega_{n}$ are the damping and frequency of the second-order model, respectively; $\delta_{T c}$ is the command signal of engine fuel equivalent ratio.

\section{Feedback Linearization Model of Hypersonic Vehicle}

The feedback linearization model is based on the differential geometrical theory as shown in [25]. The model of hypersonic 
vehicle as shown in (1) and (3) is used to control its velocity $V$ and altitude $H$, and the control inputs are its elevator angle $\delta_{z}$ and engine fuel equivalent ratio $\delta_{T c}$. Based on the differential geometrical theory, in order for the control inputs $\delta_{z}$ and $\delta_{T c}$ to appear in the model, the velocity and altitude need to be differentiated, respectively, until the control inputs whose coefficients are nonzero appear, and then the nonlinear model is quasilinearized. The argument $\mathbf{z}^{T}=\left[\begin{array}{lllll}V & \theta & \alpha & \delta_{T} & H\end{array}\right]$ is selected to differentiate $V$, and the results are as follows:

$$
\begin{aligned}
& \ddot{V}=\frac{\kappa \dot{\mathbf{z}}}{m}, \\
& \ddot{V}=\frac{\kappa \ddot{\mathbf{z}}+\dot{\mathbf{z}}^{T} K \dot{\mathbf{z}}}{m} .
\end{aligned}
$$

$\ddot{\mathbf{z}}$ appears in $V^{(3)}$ and through (1) and (3) we can get that

$$
\begin{aligned}
\ddot{\alpha}= & \dot{\omega}_{z}-\ddot{\theta}=\frac{M_{z}}{J_{z}}-\ddot{\theta} \\
= & \frac{0.5 \rho V^{2} S c\left(C_{m 0}+C_{m}^{\alpha} \alpha+C_{m}^{\delta_{T}}+\cdots\right)}{J_{z}}-\ddot{\theta} \\
& +\frac{0.5 \rho V^{2} S c C_{m}^{\delta_{z}}}{J_{z}} \delta_{z}=\ddot{\alpha}_{0}+\frac{0.5 \rho V^{2} S c C_{m}^{\delta_{z}}}{J_{z}} \delta_{z}, \\
\ddot{\delta}_{T}= & -2 \xi_{n} \omega_{n} \dot{\delta}_{T}-\omega_{n}^{2} \delta_{T}+\omega_{n}^{2} \delta_{T c}=\ddot{\delta}_{T 0}+\omega_{n}^{2} \delta_{T c} .
\end{aligned}
$$

In this way, the control input $\delta_{z}$ appears in the $\ddot{\alpha}$ expression; $\delta_{T c}$ appears in the $\ddot{\delta}_{T}$ expression; that is to say, the input control appears after the flight velocity $V$ is differentiated three times.

By the same token, the altitude $H$ is differentiated, and the results are as follows:

$$
\begin{aligned}
\ddot{H}= & \dot{V} \sin \theta+V \dot{\theta} \cos \theta, \\
H^{(3)}= & \ddot{V} \sin \theta+2 \dot{V} \dot{\theta} \cos \theta+V \ddot{\theta} \cos \theta-V \dot{\theta}^{2} \sin \theta, \\
H^{(4)}= & V^{(3)} \sin \theta+3 \ddot{V} \dot{\theta} \cos \theta+3 \dot{V} \ddot{\theta} \cos \theta-3 \dot{V} \dot{\theta}^{2} \sin \theta \\
& +V \theta^{(3)} \cos \theta-3 V \dot{\theta} \ddot{\theta} \sin \theta-V \dot{\theta}^{3} \cos \theta,
\end{aligned}
$$

where $\ddot{\theta}$ can be expressed as

$$
\begin{aligned}
\ddot{\theta} & =\frac{\pi \dot{\mathbf{z}}}{m}, \\
\theta^{(3)} & =\frac{\pi \ddot{\mathbf{z}}+\dot{\mathbf{z}}^{T} \Pi \dot{\mathbf{z}}}{m} .
\end{aligned}
$$

The expressions of various state vectors in the above equations are given in [2]. Equation (6) shows that the $V^{(3)}$ form appears in the $H^{(4)}$ expression; namely, the control inputs $\delta_{z}$ and $\delta_{T c}$ appear in the fourth-order differential expression of $H$. This shows that the hypersonic vehicle has the relative order $\rho=3+4=n$, which means that the system has no intrinsic dynamic state and thus can satisfy the essentially necessary and sufficient conditions of totalstate feedback linearization and realize the input and output feedback linearization; namely, the control outputs $V$ and $H$ can be expressed as the explicit expressions of the control inputs $\delta_{z}$ and $\delta_{T c}$.

Assuming $\ddot{\mathbf{z}}_{0}^{T}=\left[\begin{array}{lllll}\ddot{V} & \ddot{\theta} & \ddot{\alpha}_{0} & \ddot{\delta}_{T 0} & \ddot{H}\end{array}\right]$, together with (5), the dynamics equation of control output can be expressed as

$$
\begin{aligned}
V^{(3)}= & \frac{\kappa \ddot{\mathbf{z}}_{0}+\dot{\mathbf{z}}^{T} K \dot{\mathbf{z}}}{m}+b_{11} \delta_{T c}+b_{12} \delta_{z}, \\
H^{(4)}= & 3 \ddot{V} \dot{\theta} \cos \theta+3 \dot{V} \ddot{\theta} \cos \theta-3 \dot{V} \dot{\theta}^{2} \sin \theta \\
& -3 V \dot{\theta} \ddot{\theta} \sin \theta-V \dot{\theta}^{3} \cos \theta+\frac{\kappa \ddot{\mathbf{z}}_{0}+\dot{\mathbf{z}}^{T} K \dot{\mathbf{z}}}{m} \sin \theta \\
& +V \frac{\pi \ddot{\mathbf{z}}_{0}+\dot{\mathbf{z}}^{T} \Pi \dot{\mathbf{z}}}{m} \cos \theta+b_{21} \delta_{T c}+b_{22} \delta_{z} \\
\Longrightarrow & {\left[\begin{array}{c}
V^{(3)} \\
H^{(4)}
\end{array}\right]=\left[\begin{array}{c}
V_{0}^{(3)} \\
H_{0}^{(4)}
\end{array}\right]+\left[\begin{array}{ll}
b_{11} & b_{12} \\
b_{21} & b_{22}
\end{array}\right]\left[\begin{array}{c}
\delta_{T c} \\
\delta_{z}
\end{array}\right] } \\
= & F+B \mathbf{u},
\end{aligned}
$$

where

$$
\begin{aligned}
b_{11} & =\omega_{n}^{2} \frac{T^{\delta_{T}} \cos \alpha-D^{\delta_{T}}}{m}, \\
b_{12} & =\frac{\rho V^{2} S c C_{m}^{\delta_{z}}}{2 J_{z}} \frac{T^{\alpha} \cos \alpha-T \sin \alpha-D^{\alpha}}{m}, \\
b_{21} & =\omega_{n}^{2} \frac{T^{\delta_{T}} \sin (\alpha+\theta)-D^{\delta_{T}} \sin \theta+L^{\delta_{T}} \cos \theta}{m}, \\
b_{22} & =\frac{\rho V^{2} S c C_{m}^{\delta_{z}}}{2 J_{z}} \\
& \cdot \frac{T^{\alpha} \sin (\alpha+\theta)+T \cos (\alpha+\theta)-D^{\alpha} \sin \theta+L^{\alpha} \cos \theta}{m} .
\end{aligned}
$$

If $B$ is reversible, then the feedback control law is

$$
\begin{aligned}
\mathbf{u} & =\left[\begin{array}{c}
\delta_{T c} \\
\delta_{z}
\end{array}\right] \\
& =-\left[\begin{array}{ll}
b_{11} & b_{12} \\
b_{21} & b_{22}
\end{array}\right]^{-1}\left[\begin{array}{c}
V_{0}^{(3)} \\
H_{0}^{(4)}
\end{array}\right]+\left[\begin{array}{ll}
b_{11} & b_{12} \\
b_{21} & b_{22}
\end{array}\right]^{-1}\left[\begin{array}{l}
V^{(3)} \\
H^{(4)}
\end{array}\right],
\end{aligned}
$$

where $\boldsymbol{v}=\left[\begin{array}{ll}V^{(3)} & H^{(4)}\end{array}\right]^{T}$ is the new control input.

The above equation is the dynamic reverse model obtained with the precise linearization of the input and output of the hypersonic vehicle's longitudinal model (1). If the output $\mathbf{u}$ in (10) is regarded as the feedback control input of the hypersonic vehicle's longitudinal model, then its control output is (8), thereby realizing the decoupling of its altitude and velocity.

According to the flight conditions in Table 1, the influence of rudder deflection to velocity system is calculated by (8), where the engine fuel equivalent ratio is the balance value 0.3 during the cruise and changing range of the rudder is -20 degrees to 20 degrees (the results shown in Table 2). 
TABLE 1: The main technical parameters of CSULA-GHV.

\begin{tabular}{lcc}
\hline Name & Number & Unit \\
\hline Reference area $S$ & 33.4118 & $\mathrm{~m}^{2}$ \\
Average aerodynamic airfoil chord length $c$ & 33.3770 & $\mathrm{~m}$ \\
Take-off gross weight $m_{0}$ & 5000 & $\mathrm{~kg}$ \\
Rotary inertia $J_{z}$ & 460590 & $\mathrm{~kg} \cdot \mathrm{m}^{2}$ \\
Velocity $V$ & 3018 & $\mathrm{~m} / \mathrm{s}$ \\
Altitude $H$ & 30000 & $\mathrm{~m}$ \\
\hline
\end{tabular}

TABLE 2: The influence of rudder deflection to velocity system.

\begin{tabular}{ccccc}
\hline$\delta_{T c}$ & $\delta_{z}$ & $b_{12} \times \delta_{z}$ & $b_{11} \times \delta_{T c}$ & $\Delta V^{(3)}$ \\
\hline 0.3 & -20 & 45 & 3171 & 3216 \\
0.3 & 20 & 84 & 3171 & 3255 \\
\hline
\end{tabular}

TABLE 3: The influence of engine fuel equivalent ratio to altitude system.

\begin{tabular}{lcccc}
\hline$\delta_{T c}$ & $\delta_{z}$ & $b_{21} \times \delta_{T c}$ & $b_{22} \times \delta_{z}$ & $\Delta H^{(4)}$ \\
\hline 0 & 10.5 & 0 & 358 & 358 \\
0.5 & 10.5 & 48 & 513 & 561 \\
\hline
\end{tabular}

Definition 1. The upward deflection $\delta_{z}>0$ of the pitch rudder surface produces a downward steering force and the nose-up pitching moment relative to the mass center of the hypersonic vehicle.

Table 2 shows that the influence of $b_{12} \times \delta_{z}$ in velocity system is small and can be ignored.

The influence of engine fuel equivalent ratio to altitude system is calculated by (8), where the input rudder deflection is the balance value of 10.5 degrees during the cruise and changing range of the engine fuel equivalent ratio is 0 to 0.5 (the results shown in Table 3 ).

Table 3 shows that the influence of $b_{21} \times \delta_{T c}$ in altitude system is small and can be ignored. Then feedback linearization model can be described as

$$
\left[\begin{array}{l}
V^{(3)} \\
H^{(4)}
\end{array}\right]=\left[\begin{array}{l}
V_{0}^{(3)} \\
H_{0}^{(4)}
\end{array}\right]+\left[\begin{array}{cc}
b_{11} & 0 \\
0 & b_{22}
\end{array}\right]\left[\begin{array}{l}
\delta_{T c} \\
\delta_{z}
\end{array}\right]
$$

\section{Design of Backstepping Controller [26, 27]}

4.1. Velocity Control System. We use the backstepping control method to design the velocity tracking system and define $e_{V}=$ $V-V_{d}, X_{V}=\left[\begin{array}{lll}e_{V} & \dot{e}_{V} & \ddot{e}_{V}\end{array}\right]^{T}$; then velocity tracking errors can be expressed as

$$
\begin{aligned}
& \dot{X}_{V 1}=X_{V 2}, \\
& \dot{X}_{V 2}=X_{V 3}, \\
& \dot{X}_{V 3}=V^{(3)}-V_{d}^{(3)} .
\end{aligned}
$$

Based on backstepping control method, we design the controller and transform the state coordinates of each subsystem in (12) to connect the above equation with the tranquility function of a virtual control system with the known Lyapunov function.

The velocity error variable is defined as follows:

$$
\begin{aligned}
& Z_{V 1}=X_{V 1}, \\
& Z_{V 2}=X_{V 2}-\tau_{V 1}\left(X_{V 1}\right), \\
& Z_{V 3}=X_{V 3}-\tau_{V 2}\left(X_{V 1}, X_{V 2}\right),
\end{aligned}
$$

where $\tau_{V i}(i=1,2)$ is the virtual feedback. Because (13) is, in nature, a diffeomorphism, a tranquil equation (13) can guarantee a tranquil equation (12).

The Lyapunov function of $Z_{V 1}$ is defined as $W_{V 1}=$ $0.5 Z_{V 1}^{2}$. The derivation of $W_{V 1}$ produces

$$
\dot{W}_{V 1}=Z_{V 1} \dot{Z}_{V 1}=Z_{V 1} X_{V 2}=Z_{V 1}\left(Z_{V 2}+\tau_{V 1}\right) \text {. }
$$

Define $\tau_{V 1}=-\left(\Phi_{V 1}\left(\left|Z_{V 1}\right|\right)+C_{V 1}\right) Z_{V 1}\left(C_{V 1}>0\right)$; then

$$
\begin{aligned}
\dot{Z}_{V 1} & =Z_{V 2}+\tau_{V 1} \\
& =-\left(\Phi_{V 1}\left(\left|Z_{V 1}\right|\right)+C_{V 1}\right) Z_{V 1}+Z_{V 2} .
\end{aligned}
$$

The derivation of $W_{V 1}$ produces

$$
\dot{W}_{V 1}=-\left(\Phi_{V 1}\left(\left|Z_{V 1}\right|\right)+C_{V 1}\right) Z_{V 1}^{2}+Z_{V 1} Z_{V 2}
$$

where $\Phi_{V 1}(\cdot)$ is the designed $K$ class function [11]. $\Phi_{V 1}\left(\left|Z_{V 1}\right|\right)=(1 / 3) \varepsilon_{V 1} Z_{V 1}^{2}$ and $\varepsilon_{V 1}>0$ are selected as design parameters.

The Lyapunov functions of $Z_{V 1}$ and $Z_{V 2}$ are defined as $W_{V 2}=0.5 Z_{V 1}^{2}+0.5 Z_{V 2}^{2}$. Given $\dot{Z}_{V 2}=X_{V 3}+J_{V 2}$, the derivation of $W_{V 2}$ produces

$$
\begin{aligned}
\dot{W}_{V 2}= & -\left(\Phi_{V 1}\left(\left|Z_{V 1}\right|\right)+C_{V 1}\right) Z_{V 1}^{2} \\
& +Z_{V 2}\left(Z_{V 1}+X_{V 3}+J_{V 2}\right),
\end{aligned}
$$

where

$$
J_{V 2}=-\frac{\partial \tau_{V 1}}{\partial Z_{V 1}} \dot{Z}_{V 1}=-\left(\varepsilon_{V 1} Z_{V 1}^{2}+C_{V 1}\right) \dot{Z}_{V 1}
$$

Define $\tau_{V 2}=-\left(\Phi_{V 2}\left(\left|Z_{V 2}\right|\right)+C_{V 2}\right) Z_{V 2}-Z_{V 1}-J_{V 2}\left(C_{V 2}>\right.$ $0)$ and $X_{V 3}=Z_{V 3}+\tau_{V 2}$; then

$$
\begin{aligned}
\dot{Z}_{V 2}= & -Z_{V 1}-\left(\Phi_{V 2}\left(\left|Z_{V 2}\right|\right)+C_{V 2}\right) Z_{V 2}+Z_{V 3}, \\
\dot{W}_{V 2}= & -\left(\Phi_{V 1}\left(\left|Z_{V 1}\right|\right)+C_{V 1}\right) Z_{V 1}^{2} \\
& -\left(\Phi_{V 2}\left(\left|Z_{V 2}\right|\right)+C_{V 2}\right) Z_{V 2}^{2}+Z_{V 2} Z_{V 3} .
\end{aligned}
$$

$\Phi_{V 2}\left(\left|Z_{V 2}\right|\right)=(1 / 3) \varepsilon_{V 2} Z_{V 2}^{2}$ and $\varepsilon_{V 2}>0$ are selected as design parameters.

The Lyapunov functions of $Z_{V 1}, Z_{V 2}$, and $Z_{V 3}$ are defined as $W_{V 3}=0.5 Z_{V 1}^{2}+0.5 Z_{V 2}^{2}+0.5 Z_{V 3}^{2}$. The derivation of $W_{V 3}$ produces

$$
\begin{aligned}
\dot{W}_{V 3}= & -\left(\Phi_{V 1}\left(\left|Z_{V 1}\right|\right)+C_{V 1}\right) Z_{V 1}^{2} \\
& -\left(\Phi_{V 2}\left(\left|Z_{V 2}\right|\right)+C_{V 2}\right) Z_{V 2}^{2}+Z_{V 2} Z_{V 3} \\
& +Z_{V 3} \dot{Z}_{V 3} .
\end{aligned}
$$


Define $\dot{Z}_{V 3}=-\left(\Phi_{V 3}\left(\left|Z_{V 3}\right|\right)+C_{V 3}\right) Z_{V 3}-Z_{V 2}\left(C_{V 3}>0\right)$; then

$$
\begin{aligned}
\dot{W}_{V 3}= & -\left(\Phi_{V 1}\left(\left|Z_{V 1}\right|\right)+C_{V 1}\right) Z_{V 1}^{2} \\
& -\left(\Phi_{V 2}\left(\left|Z_{V 2}\right|\right)+C_{V 2}\right) Z_{V 2}^{2} \\
& -\left(\Phi_{V 3}\left(\left|Z_{V 3}\right|\right)+C_{V 3}\right) Z_{V 3}^{2}<0 .
\end{aligned}
$$

$\Phi_{V 3}\left(\left|Z_{V 3}\right|\right)=(1 / 3) \varepsilon_{V 3} Z_{V 3}^{2}$ and $\varepsilon_{V 3}>0$ are selected as design parameters.

Therefore, (13) for velocity error tracking system has the Lyapunov stability. Define $\dot{Z}_{V 3}=\dot{X}_{V 3}+J_{V 3}$; then

$$
\begin{aligned}
V^{(3)} & =V_{d}^{(3)}+\dot{Z}_{V 3}-J_{V 3} \\
& =V_{d}^{(3)}-Z_{V 2}-\left(\Phi_{V 3}\left(\left|Z_{V 3}\right|\right)+C_{V 3}\right) Z_{V 3}-J_{V 3} .
\end{aligned}
$$

4.2. Altitude Control System. Applying the adaptive backstepping control law to the altitude system with actuator constraints, we define $X_{H 1}=H, X_{H 2}=\dot{H}, X_{H 3}=\ddot{H}$, and $X_{H 4}=\dddot{H}$, thus obtaining the altitude control system:

$$
\begin{aligned}
& \dot{X}_{H 1}=X_{H 2}, \\
& \dot{X}_{H 2}=X_{H 3}, \\
& \dot{X}_{H 3}=X_{H 4}, \\
& \dot{X}_{H 4}=H^{(4)} .
\end{aligned}
$$

We have $H^{(4)}$ in (11), considering that only $b_{22}$ has uncertainty in the altitude system and assuming $f_{H 4}=H_{0}^{(4)}+$ $\left(b_{21} \delta_{T c}\right)_{0}$.

Step 1. Define $Z_{H 1}=X_{H 1}-H_{d}, Z_{H 2}=X_{H 2}-\phi_{H 1}$ and take $\phi_{H 1}$ as the virtual control of $X_{H 2}$.

The Lyapunov function is selected as $W_{H 1}=0.5 Z_{H 1}^{2}$. The derivative of $W_{H 1}$ is

$$
\dot{W}_{H 1}=Z_{H 1} \dot{Z}_{H 1}=Z_{H 1}\left(Z_{H 2}+\phi_{H 1}-\dot{H}_{d}\right) \text {. }
$$

The virtual control is selected as $\phi_{H 1}=\dot{H}_{d}-C_{H 1} Z_{H 1}$, and we obtain

$$
\dot{W}_{H 1}=-C_{H 1} Z_{H 1}^{2}+Z_{H 1} Z_{H 2} \text {. }
$$

Step 2. Define $Z_{H 3}=X_{H 3}-\phi_{H 2}$, and take $\phi_{H_{2}}$ as the virtual control of $X_{\mathrm{H} 3}$.

The Lyapunov function is selected as $W_{H 2}=W_{H 1}+$ $0.5 Z_{\mathrm{H} 2}^{2}$. The derivative of $W_{\mathrm{H} 2}$ is

$$
\begin{aligned}
\dot{W}_{H 2} & =\dot{W}_{H 1}+Z_{H 2} \dot{Z}_{H 2} \\
& =-C_{H 1} Z_{H 1}^{2}+Z_{H 2}\left(Z_{H 1}+Z_{H 3}+\phi_{H 2}-\dot{\phi}_{H 1}\right) .
\end{aligned}
$$

The virtual control is selected as $\phi_{H 2}=\dot{\phi}_{H 1}-Z_{H 1}-$ $\mathrm{C}_{\mathrm{H} 2} \mathrm{Z}_{\mathrm{H} 2}$, and we obtain

$$
\dot{W}_{H 2}=-C_{H 1} Z_{H 1}^{2}-C_{H 2} Z_{H 2}^{2}+Z_{H 2} Z_{H 3} .
$$

Step 3. Define $Z_{H 4}=X_{H 4}-\phi_{H 3}$, and take $\phi_{H 3}$ as the virtual control of $X_{H 4}$.

The Lyapunov function is selected as $W_{H 3}=W_{H_{2}}+$ $0.5 Z_{H 3}^{2}$. The derivative of $W_{H 3}$ is

$$
\begin{aligned}
\dot{W}_{H 3}= & \dot{W}_{H 2}+Z_{H 3} \dot{Z}_{H 3} \\
= & -C_{H 1} Z_{H 1}^{2}-C_{H 2} Z_{H 2}^{2} \\
& +Z_{H 3}\left(Z_{H 2}+Z_{H 4}+\phi_{H 3}-\dot{\phi}_{H 2}\right) .
\end{aligned}
$$

The virtual control is selected as $\phi_{H_{3}}=\dot{\phi}_{H_{2}}-Z_{H_{2}}-$ $\mathrm{C}_{\mathrm{H} 3} Z_{\mathrm{H} 3}$, and we obtain

$$
\dot{W}_{H 3}=-C_{H 1} Z_{H 1}^{2}-C_{H 2} Z_{H 2}^{2}-C_{H 3} Z_{H 3}^{2}+Z_{H 3} Z_{H 4} .
$$

Step 4. The Lyapunov function is selected as $W_{H 4}=W_{H 3}+$ $0.5 Z_{H 4}^{2}+0.5 \Gamma^{-1} \widetilde{b}_{22}^{2}$. The derivative of $W_{H 4}$ is

$$
\begin{aligned}
\dot{W}_{H 4}= & \dot{W}_{H 3}+Z_{H 4} \dot{Z}_{H 4}-\Gamma^{-1}\left(b_{22}-\widehat{b}_{22}\right) \dot{\widehat{b}}_{22}, \\
\dot{W}_{H 4}= & -\sum_{i=1}^{3} C_{H i} Z_{H i}^{2} \\
& +Z_{H 4}\left(Z_{H 3}+f_{H 4}+b_{22} \delta_{z}-\dot{\phi}_{H 3}\right) \\
& -\Gamma^{-1}\left(b_{22}-\widehat{b}_{22}\right) \dot{\widehat{b}}_{22} .
\end{aligned}
$$

The command of elevator angle is selected as

$$
\delta_{z}=\frac{\left(-C_{H 4} Z_{H 4}-Z_{H 3}-f_{H 4}+\dot{\phi}_{H 3}\right)}{\hat{b}_{22}} .
$$

Introducing (32) into (31), we have

$$
\begin{aligned}
\dot{W}_{H 4}= & -\sum_{i=1}^{3} C_{H i} Z_{H i}^{2}+Z_{H 4}\left(\left(b_{22}-\widehat{b}_{22}\right) \delta_{z}-C_{H 4} Z_{H 4}\right) \\
& -\Gamma^{-1}\left(b_{22}-\widehat{b}_{22}\right) \dot{\hat{b}}_{22} \\
\dot{W}_{H 4}= & -\sum_{i=1}^{4} C_{H i} Z_{H i}^{2} \\
& -\Gamma^{-1}\left(b_{22}-\widehat{b}_{22}\right)\left(\dot{\hat{b}}_{22}-\Gamma Z_{H 4} \delta_{z}\right) .
\end{aligned}
$$

The adaptive law of the estimated parameter is modified as

$$
\dot{\hat{b}}_{22}=\Gamma Z_{H 4} \delta_{z}
$$

where $\dot{W}_{H 4} \leq 0$; according to the Lyapunov stability analysis the altitude system is asymptotically stable.

4.3. Actuator Constraint Compensation. The objective of this section is to ensure stability when actuator constraints are in effect. The actuator system is shown in Figure 1. The filter [28] generates the command and command derivative while enforcing magnitude constraint. In the figure, $\delta_{z c}$ is 


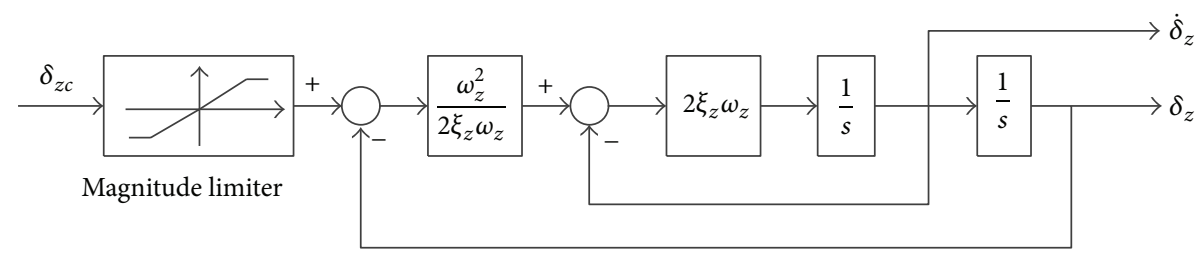

FIGURE 1: The filter enforcing magnitude constraint.

the elevator angle command; $\delta_{z}$ is the actual output of the filter; $\omega_{z}$ is the bandwidth of the actuator, and $\xi_{z}$ is the damping coefficient.

We introduce the tracking error of input and output in the filter into the control law, based on which we obtain the altitude adaptive law and the actuator command with magnitude constraints.

Define $\widetilde{Z}_{H 4}=Z_{H 4}-e_{4}$, where

$$
\dot{e}_{4}=-C_{H 4} e_{4}-\widehat{b}_{22}\left(\delta_{z c}-\delta_{z}\right) .
$$

In Step 3, the derivative of the Lyapunov function $W_{H 3}$ is

$$
\begin{aligned}
\dot{W}_{H 3}= & -C_{H 1} Z_{H 1}^{2}-C_{H 2} Z_{H 2}^{2} \\
& +Z_{H 3}\left(Z_{H 2}+\widetilde{Z}_{H 4}+e_{4}+\phi_{H 3}-\dot{\phi}_{H 2}\right) .
\end{aligned}
$$

The virtual control is selected as $\phi_{\mathrm{H} 3}=\dot{\phi}_{\mathrm{H} 2}-Z_{\mathrm{H} 2}-$ $\mathrm{C}_{\mathrm{H} 3} Z_{\mathrm{H} 3}-e_{4}$, and we have

$$
\dot{W}_{H 3}=-C_{H 1} Z_{H 1}^{2}-C_{H 2} Z_{H 2}^{2}-C_{H 3} Z_{H 3}^{2}+Z_{H 3} \widetilde{Z}_{H 4} .
$$

According to (32), the actuator command is selected as

$$
\delta_{z c}=\frac{\left(-C_{H 4} Z_{H 4}-Z_{H 3}-f_{H 4}+\dot{\phi}_{H 3}\right)}{\hat{b}_{22}},
$$

where

$$
\begin{aligned}
& \dot{Z}_{H 4}=\dot{X}_{H 4}-\dot{\phi}_{H 3}=b_{22} \delta_{z}-C_{H 4} Z_{H 4}-\widehat{b}_{22} \delta_{z c}-Z_{H 3}, \\
& \dot{\bar{Z}}_{H 4}=\dot{Z}_{H 4}-\dot{e}_{4}=-C_{H 4} \widetilde{Z}_{H 4}+\widetilde{b}_{22} \delta_{z}-Z_{H 3} .
\end{aligned}
$$

Redefine the Lyapunov function in Step 4 as $W_{H 4}^{\prime}=W_{H 3}+$ $0.5 \widetilde{Z}_{H 4}^{2}+0.5 \Gamma^{-1} \widetilde{b}_{22}^{2}$, and the derivative of $W_{H 4}^{\prime}$ is

$$
\begin{aligned}
\dot{W}_{H 4}^{\prime}= & -\sum_{i=1}^{3} C_{H i} Z_{H i}^{2}-C_{H 4} \widetilde{Z}_{H 4}^{2} \\
& -\Gamma^{-1} \widetilde{b}_{22}\left(\dot{\hat{b}}_{22}-\Gamma \widetilde{Z}_{H 4} \delta_{z}\right) .
\end{aligned}
$$
as

$$
\dot{\hat{b}}_{22}=\Gamma \widetilde{Z}_{H 4} \delta_{z}
$$

where $\dot{W}_{H 4}^{\prime} \leq 0$; according to the Lyapunov stability analysis the altitude system is asymptotically stable.
4.4. The Arbitrary-Order Robust Exact Differentiator. The main difficulty for the higher-order differentiator is the obvious differentiation sensitivity to input noise. We use the arbitrary-order robust exact differentiator [29] designed by Levant to differentiate the velocity command and the altitude command in the feedback linearization $V^{(3)}$ and $H^{(4)}$. This differentiator [30] possesses the important features of finite-time exact convergence to the real values of estimated derivatives and the possibility to provide some degree of smoothness to the estimated signal.

We use $z_{i}$ and $v_{i}$ to denote the scalar differentiator variables. If a certain signal $f(t)$ is an $r$ th order derivative, then $f^{(n-1)}(t)$ has a definite Lipschitz constant $L>0$, thus having the following formulae for the differentiator of the $n$ number of orders:

$$
\begin{aligned}
& \dot{z}_{0}=v_{0}, \\
& v_{0}=-\lambda_{0} L^{1 /(n+1)}\left|z_{0}-f(t)\right|^{n /(n+1)} \operatorname{sign}\left(z_{0}-f(t)\right)+z_{1}, \\
& \dot{z}_{1}=v_{1}, \\
& v_{1}=-\lambda_{1} L^{2 /(n+1)}\left|z_{1}-v_{0}\right|^{(n-1) / n} \operatorname{sign}\left(z_{1}-v_{0}\right)+z_{2}, \\
& \vdots \\
& \dot{z}_{n-1}=v_{n-1}, \\
& v_{n-1}=-\lambda_{n-1} L^{1 / 2}\left|z_{n-1}-v_{n-2}\right|^{1 / 2} \operatorname{sign}\left(z_{n-1}-v_{n-2}\right)+z_{n}, \\
& \dot{z}_{n}=-\lambda_{n} L \operatorname{sign}\left(z_{n}-v_{n-1}\right) .
\end{aligned}
$$

If $\lambda_{i}>0$ is sufficiently large, the convergence is guaranteed.

We take the velocity system as an example, and the altitude system has the same form. The 3rd-order differentiator built according to the following parameters and equations are used in the velocity system. The initial value $\lambda_{0}=\lambda_{1}=\lambda_{2}=$ $\lambda_{3}=50, z_{0}=0.1$; another initial value is $z_{1}=z_{2}=z_{3}=0$; $L=1400$, and the simulation step is 0.001 seconds. The altitude system has the same form:

$$
\begin{aligned}
& \dot{z}_{0}=v_{0}, \quad v_{0}=-\lambda_{0} L^{1 / 4}\left|z_{0}-\dot{q}\right|^{3 / 4} \operatorname{sign}\left(z_{0}-\dot{q}\right)+z_{1}, \\
& \dot{z}_{1}=v_{1}, \\
& v_{1}=-\lambda_{1} L^{1 / 3}\left|z_{1}-v_{0}\right|^{2 / 3} \operatorname{sign}\left(z_{1}-v_{0}\right)+z_{2},
\end{aligned}
$$



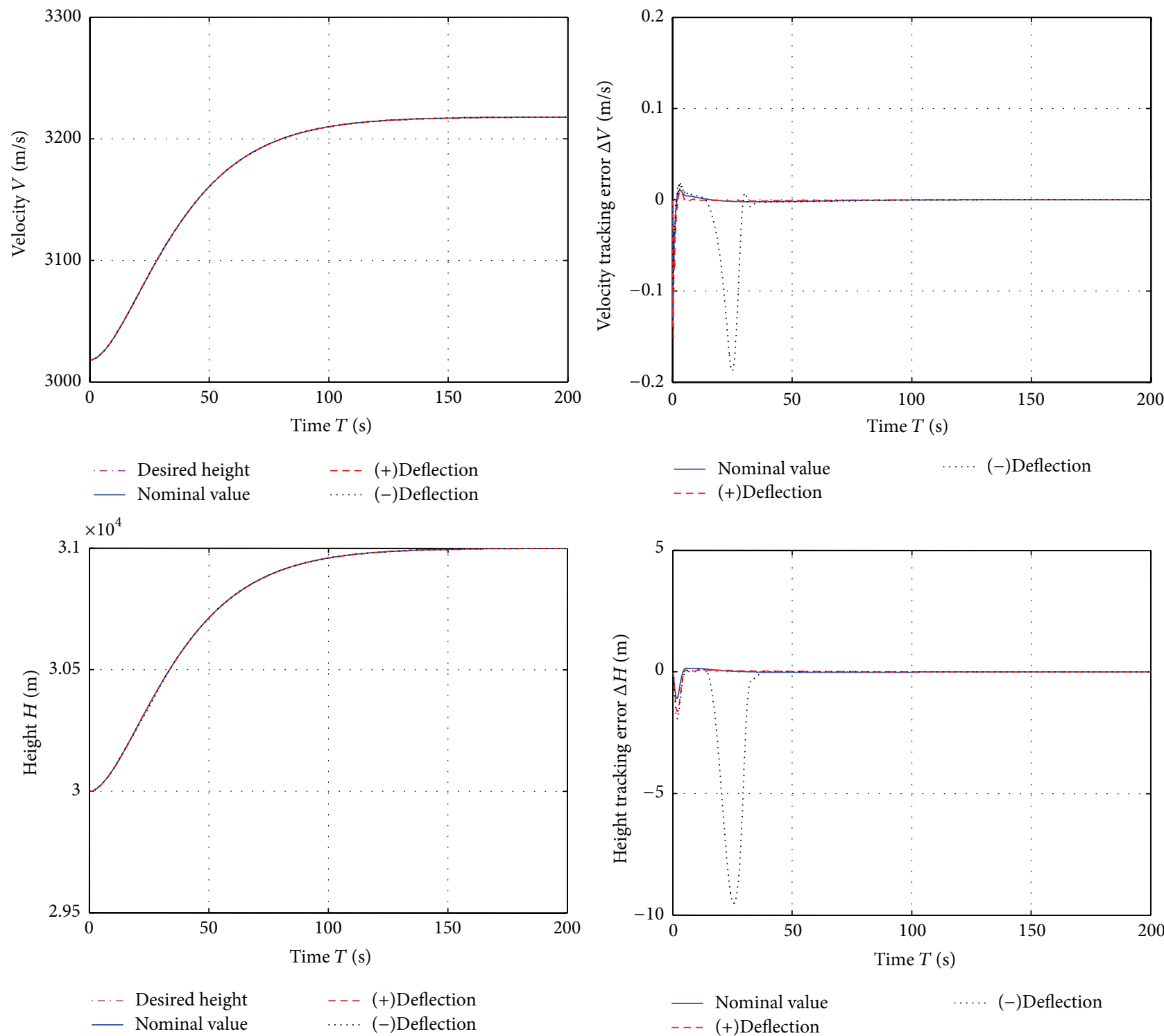

-.- Desired height

--- (+)Deflection

…. (-)Deflection

- - (+)Deflection

FIGURE 2: Response to tracking commands and tracking error curves.

$\dot{z}_{2}=v_{2}$

The control parameters are selected as follows:

$$
\begin{gathered}
v_{2}=-\lambda_{2} L^{1 / 2}\left|z_{2}-v_{1}\right|^{1 / 2} \operatorname{sign}\left(z_{2}-v_{1}\right)+z_{3}, \\
\dot{z}_{3}=-\lambda_{3} L \operatorname{sign}\left(z_{3}-v_{2}\right),
\end{gathered}
$$

where $z_{3}, z_{2}, z_{1}$, and $z_{0}$ are the estimations of $V^{(3)}, V^{(2)}, V^{(1)}$, and $V$.

\section{Simulations and Analysis}

5.1. The Deflection Simulation of the Adaptive Backstepping Control Method. The deflections and deflection values are given in Table 4.

$$
\begin{aligned}
& C_{V 1}=2, \\
& C_{V 2}=17, \\
& C_{V 3}=11, \\
& \varepsilon_{V 1}=0.1, \\
& \varepsilon_{V 2}=0.001, \\
& \varepsilon_{V 3}=0.01, \\
& C_{H 1}=0.05, \\
& C_{H 2}=2,
\end{aligned}
$$


TABLE 4: The deflections and deflection values.

\begin{tabular}{lc}
\hline Deflections & Deflection values \\
\hline Lift $\Delta L$ & $\pm 5 \%$ \\
Pitch moment $\Delta M_{z}$ & $\pm 20 \%$ \\
Atmospheric density $\Delta \rho$ & $\pm 5 \%$ \\
Rotary inertia $\Delta J_{z}$ & $\pm 10 \%$ \\
Average aerodynamic airfoil chord length $\Delta c$ & $\pm 10 \%$ \\
Total length of the flight vehicle $\Delta L_{\text {ref }}$ & $\pm 10 \%$ \\
Take-off gross weight $\Delta m_{0}$ & $\pm 10 \%$ \\
\hline
\end{tabular}

$$
\begin{aligned}
C_{H 3} & =10, \\
C_{H 4} & =2, \\
\Gamma & =0.01 .
\end{aligned}
$$

The command signals are hypersonic vehicle's altitude increases $1000 \mathrm{~m}$ and velocity increases $200 \mathrm{~m} / \mathrm{s}$, and these tracking command signals are joined through the following filter shown in (45):

$$
P(s)=\frac{0.05^{2}}{s^{2}+2 \times 1 \times 0.05 s+0.05^{2}} .
$$

The simulation results with backstepping controller are given in Figures 2 and 3.

The simulation results show that, in its design process, the backstepping control method based on feedback linearization selects the feedback gains pertinent to errors and has a very small tracking error. Its velocity tracking error is within $0.15 \mathrm{~m} / \mathrm{s}$ and the altitude tracking error is within $1 \mathrm{~m}$ throughout the flight. Meanwhile, all the state variables of the hypersonic vehicle can quickly approach their own stable values; the change in its angle of attack is within $-0.5^{\circ}$ to $-1^{\circ}$; thus its flight trajectory and attitude are coordinated. The deflection comparison results show that the backstepping control method can well suppress the uncertainties of structural parameters and aerodynamic parameters in the model of hypersonic vehicle and track its velocity and altitude, tracking errors being within the tolerable range, and that eventually all the state variables can reach their steady-state values.

5.2. Comparison with LQR Control Law. According to [6, 7], based on Section 3, we combine feedback linearization model with LQR control theory and design appropriate LQR control algorithm for altitude and velocity system. The control parameters are selected as follows:

$$
\begin{aligned}
& Q_{V}=\operatorname{diag}\left\{\begin{array}{lll}
0.1 & 10 & 0.6
\end{array},\right. \\
& R_{V}=1.5, \\
& Q_{H}=\operatorname{diag}\left\{\begin{array}{llll}
0.15 & 4 & 30 & 5
\end{array}\right\}, \\
& R_{H}=1.2 .
\end{aligned}
$$
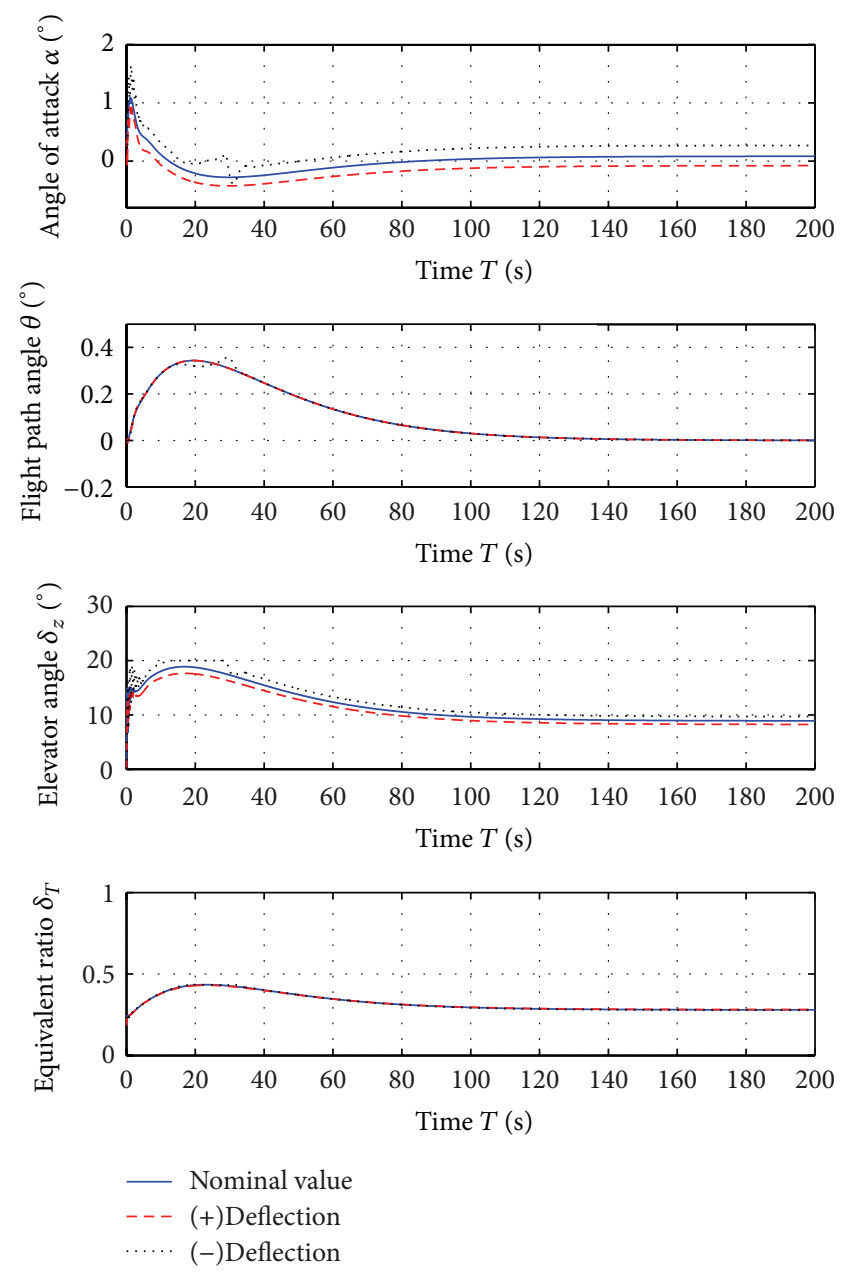

FIgURE 3: Attitude response curves.

Two sets of feedback gains are shown as follows:

$$
\begin{aligned}
& K_{V}=\left[\begin{array}{llll}
-0.2582 & -2.8169 & -2.4564
\end{array}\right], \\
& K_{H}=\left[\begin{array}{llll}
-0.3536 & -2.8762 & -6.9850 & -4.2587
\end{array}\right] .
\end{aligned}
$$

Consider the following interferences, such as gust, engine mounting deviation, wing mounting deviation, centroid deviation, and control system error. Each interference aforementioned can be converted to an equivalent additional rudder deflection, we assume that those interferences occur at 150 $s$ from the beginning of the simulation (maximum deviation angle is 5 degrees and lasts for 10 seconds). Comparing the classical LQR control law and the adaptive backstepping control law with actuator constraints proposed in this paper, the results are shown in Figures 4 and 5.

The simulation results show that the classical LQR control law and the adaptive backstepping control law with rudder system constraints based on feedback linearization have the same response characteristics for altitude and velocity command. However, after introducing interferences, tracking capability of LQR control law is poor, angle of attack changes intensely, which cannot meet the scramjet engine operating conditions, and large rudder deflection angle and engine 

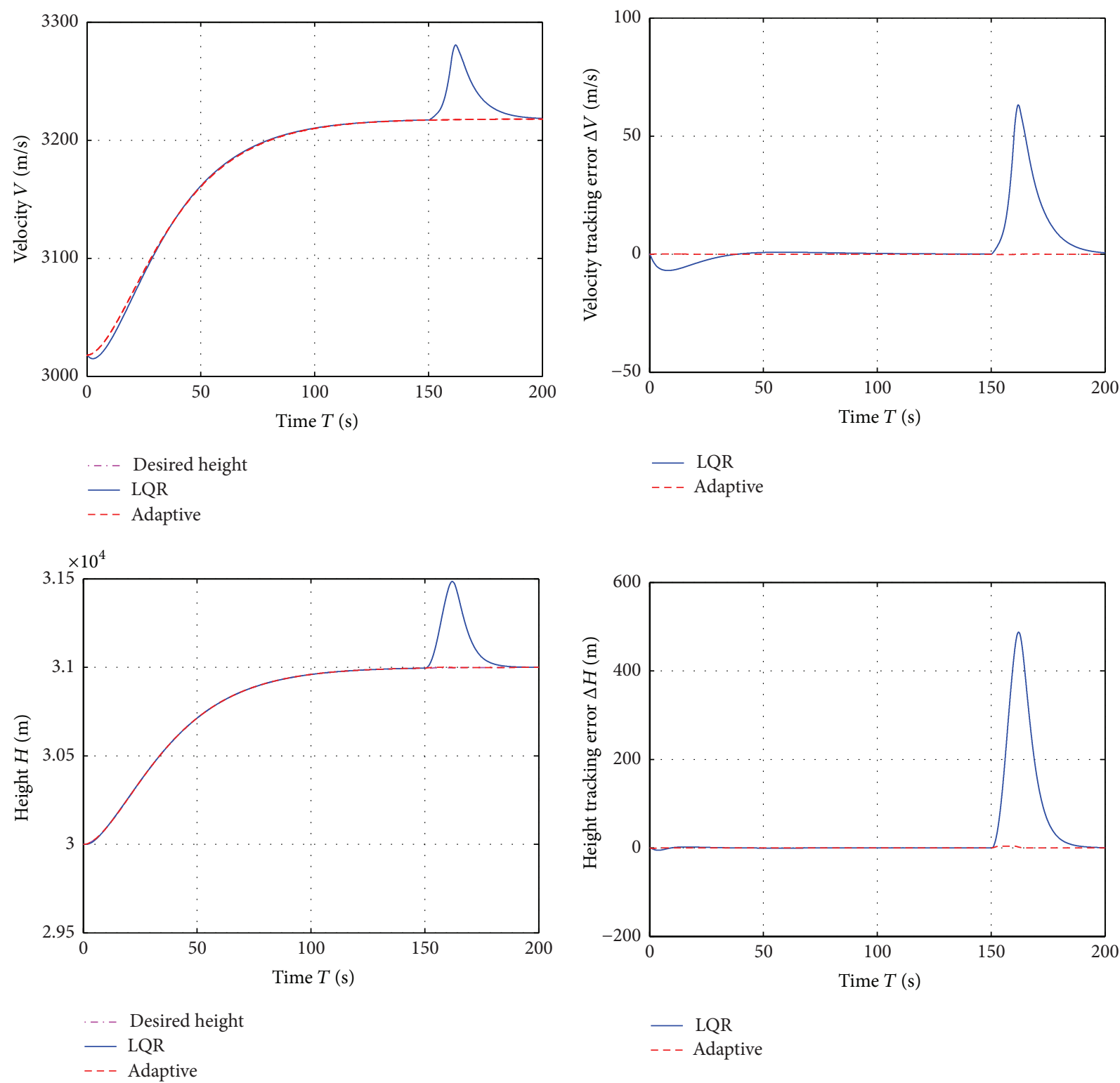

FIGURE 4: Tracking error curves with interferences.

fuel equivalent ratio are required to suppress the influence of interference. The control law proposed in this paper is insensitive to interferences, altitude and velocity control system can track the command signals quickly and exactly and have the ability to suppress interferences, and only small shake of angle of attack emerges, which does not affect the normal operation of scramjet engine; rudder deflection angle and fuel equivalent ratio are changing within a reasonable range; therefore, the new designed controller has strong robustness.

\section{Conclusion}

The distinct strong coupling between the airframe and the engine of a hypersonic vehicle and its large envelope flight characteristics create troubles for its altitude and velocity control. The paper uses the feedback linearization method to quasilinearize the nonlinear model and then the adaptive backstepping control with actuator constraints is designed as the outer-loop auxiliary controller. The simulation results show that, under the circumstance of parametric uncertainty and interferences, the hypersonic vehicle can steadily track its altitude and velocity commands signals with a quick speed. There is no steady-state error and tracking error is relatively small. All state parameters change within a reasonable range and reach a steady state value finally. When the hypersonic vehicle receives interferences, the new controller is insensitive to interferences and shows strong robustness. It can ensure the stability of angle of attack, and rudder deflection angle and fuel equivalent ratio change within a reasonable range. 

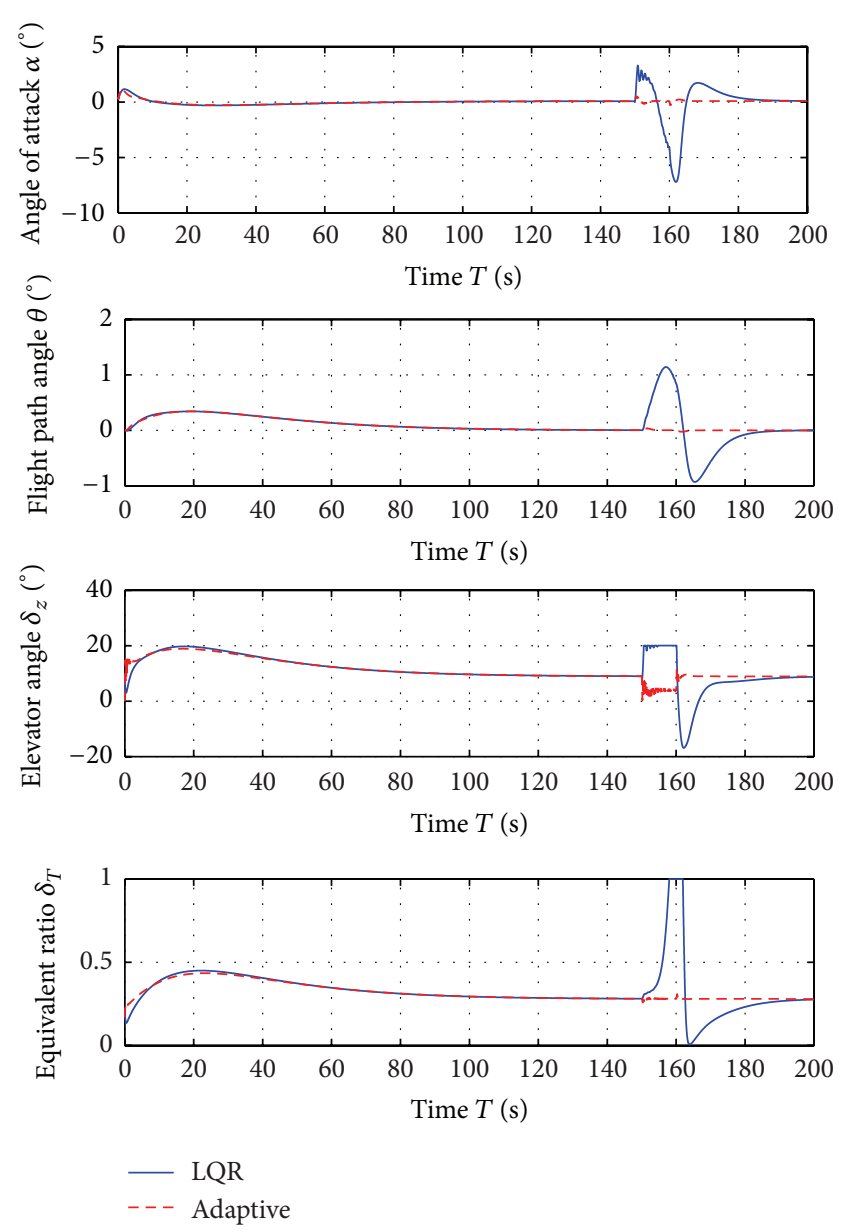

FIGURE 5: Attitude response curves with interferences.

\section{Conflict of Interests}

The authors declare that there is no conflict of interests regarding the publication of this paper.

\section{Acknowledgments}

This work was partly supported by the Natural Science Foundation of China (Grant no. 91216104) and the Fundamental Research Foundation of Northwestern Polytechnical University (Grant no. JC20110238).

\section{References}

[1] B. Xu and Z. Shi, "An overview on flight dynamics and control approaches for hypersonic vehicles," Science China Information Sciences, vol. 58, no. 7, Article ID 070201, 19 pages, 2015.

[2] Q. Wang and R. F. Stengel, "Robust nonlinear control of a hypersonic aircraft," Journal of Guidance, Control, and Dynamics, vol. 23, no. 4, pp. 577-585, 2000.

[3] A. N. Atassi and H. K. Khalil, "A separation principle for the control of a class of nonlinear systems," IEEE Transactions on Automatic Control, vol. 46, no. 5, pp. 742-746, 2001.

[4] D. O. Sigthorsson, P. Jankovsky, A. Serrani, S. Yurkovich, M. A. Bolender, and D. B. Doman, "Robust linear output feedback control of an airbreathing hypersonic vehicle," Journal of Guidance, Control, and Dynamics, vol. 31, no. 4, pp. 10521066, 2008.

[5] P. Jankovsky, D. O. Sigthorsson, A. Serrani, S. Yurkovich, M. A. Bolender, and D. B. Doman, "Output feedback control and sensor placement for a hypersonic vehicle model," in Proceedings of the AIAA Guidance, Navigation, and Control Conference, pp. 247-268, August 2007.

[6] O. U. Rehman, B. Fidan, and I. Petersen, "Uncertainty modeling and robust minimax LQR control of hypersonic flight vehicles," in Proceedings of the AIAA Guidance, Navigation, and Control Conference, pp. 1-15, AIAA, Toronto, Canada, August 2010.

[7] O. U. Rehman, I. R. Petersen, and B. Fidan, "Robust nonlinear control of a nonlinear uncertain system with input coupling and its application to hypersonic flight vehicles," in Proceedings of the IEEE International Conference on Control Applications (CCA '10), pp. 1451-1457, IEEE, Yokohama, Japan, September 2010.

[8] H. Sun, S. Li, and C. Sun, "Robust adaptive integral-slidingmode fault-tolerant control for airbreathing hypersonic vehicles," Proceedings of the Institution of Mechanical Engineers Part I: Journal of Systems and Control Engineering, vol. 226, no. 10, pp. 1344-1355, 2012.

[9] Z. Hongmei, Hypersonic Flight Vehicle Modeling and Control, Tianjin University, Tianjin, China, 2011.

[10] A. Hao, Exploring hypersonic flight vehicle modeling and control method [M.S. thesis], Harbin University, Harbin, China, 2013.

[11] D. Gao and Z. Sun, "Discrete and fuzzy adaptive control of hypersonic flight vehicle," Space Control Techniques and Their Application, no. 5, pp. 13-19, 2009.

[12] D.-X. Gao, Z.-Q. Sun, X. Luo, and T.-R. Du, "Fuzzy adaptive control for hypersonic vehicle via backstepping method," Control Theory and Applications, vol. 25, no. 5, pp. 805-810, 2008.

[13] Z. Jianfei, F. Yong, Z. Xuemei, and Y. Xuqiang, "Reverse terminal sliding mode control of uncertain nonlinear system," Control Theory and Decision Making, no. 4, pp. 410-414, 2009.

[14] B. Xu, F. Sun, C. Yang, D. Gao, and J. Ren, "Adaptive discretetime controller design with neural network for hypersonic flight vehicle via back-stepping," International Journal of Control, vol. 84, no. 9, pp. 1543-1552, 2011.

[15] B. Xu, F. Sun, H. Liu, and J. Ren, "Adaptive Kriging controller design for hypersonic flight vehicle via back-stepping," IET Control Theory \& Applications, vol. 6, no. 4, pp. 487-497, 2012.

[16] B. Xu, C. Yang, and Y. Pan, "Global neural dynamic surface tracking control of strict-feedback systems with application to hypersonic flight vehicle," IEEE Transactions on Neural Networks and Learning Systems, 2015.

[17] B. Xu, Z. Shi, C. Yang, and F. Sun, "Composite neural dynamic surface control of a class of uncertain nonlinear systems in strict-feedback form," IEEE Transactions on Cybernetics, vol. 44, no. 12, pp. 2626-2634, 2014.

[18] H. Xu, M. D. Mirmirani, and P. A. Ioannou, "Adaptive sliding mode control design for a hypersonic flight vehicle," Journal of Guidance, Control, and Dynamics, vol. 27, no. 5, pp. 829-838, 2004.

[19] J. Farrell, M. Sharma, and M. Polycarpou, "Backstepping-based flight control with adaptive function approximation," Journal of Guidance, Control, and Dynamics, vol. 28, no. 6, pp. 1089-1102, 2005.

[20] J. Farrell, M. Polycarpou, and M. Sharma, "On-line approximation based control of uncertain nonlinear systems with magnitude, rate and bandwidth constraints on the states and 
actuators," in Proceedings of the American Control Conference (AAC '04), pp. 2557-2562, Boston, Mass, USA, July 2004.

[21] J. Farrell, M. Polycarpou, and M. Sharma, "Adaptive backstepping with magnitude, rate, and bandwidth constraints: aircraft longitude control," in Proceedings of the American Control Conference, pp. 3898-3904, Denver, Colo, USA, June 2003.

[22] K. P. Tee, S. S. Ge, and E. H. Tay, "Barrier Lyapunov functions for the control of output-constrained nonlinear systems," Automatica, vol. 45, no. 4, pp. 918-927, 2009.

[23] A. D. Clark, M. D. Mirmirani, C. Wu, S. Choi, and M. Kuipers, "An aero-propulsion integrated elastic model of a generic airbreathing hypersonic vehicle," in Proceedings of the AIAA Guidance, Navigation, and Control Conference, pp. 3699-3718, August 2006.

[24] A. Clark and C. Wu, "Development of an airframe-propulsion integrated generic hypersonic vehicle model," in Proceedings of the AIAA Aerospace Science Meeting and Exhibit, pp. 1-13, Reno, Nev, USA, January 2006.

[25] D. Li, Nonlinear Control System, Northwestern Polytechnical University Press, Xi'an, China, 2009.

[26] Z. Yanming, Nonlinear Control of Hypersonic Flight Vehicle, Northeast University, Shenyang, China, 2009.

[27] Y. Liu and Y. Lu, "Longitudinal and reverse flight control of hypersonic vehicle based on backstepping control method," Control Theory and Decision Making, vol. 22, no. 3, pp. 313-317, 2007.

[28] B. Xu, X. Huang, D. Wang, and F. Sun, "Dynamic surface control of constrained hypersonic flight models with parameter estimation and actuator compensation," Asian Journal of Control, vol. 16, no. 1, pp. 162-174, 2014.

[29] A. Levant, "Higher-order sliding modes, differentiation and output-feedback control," International Journal of Control, vol. 76, no. 9-10, pp. 924-941, 2003.

[30] J. Davila and G. Carlo, "Attitude control of spacecraft using robust backstepping controller based on high order sliding modes," in Proceedings of the Guidance, Navigation, and Control and Co-Located Conferences, AIAA 2013-5121, Boston, Mass, USA, April 2013. 

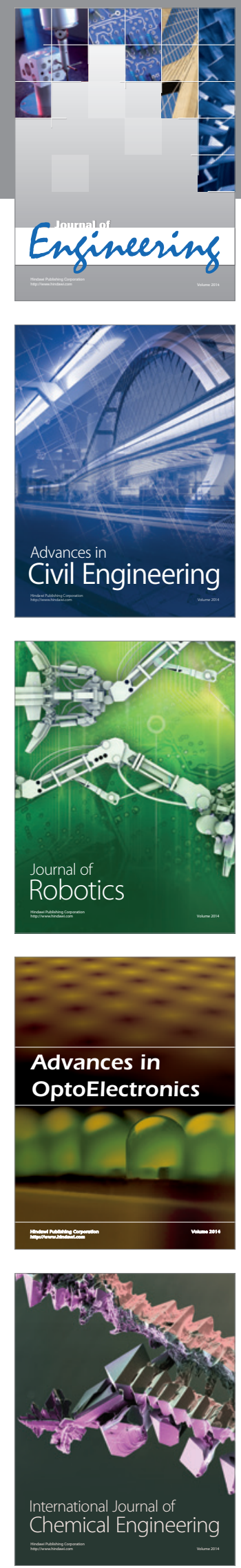

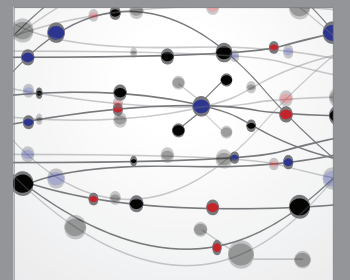

The Scientific World Journal
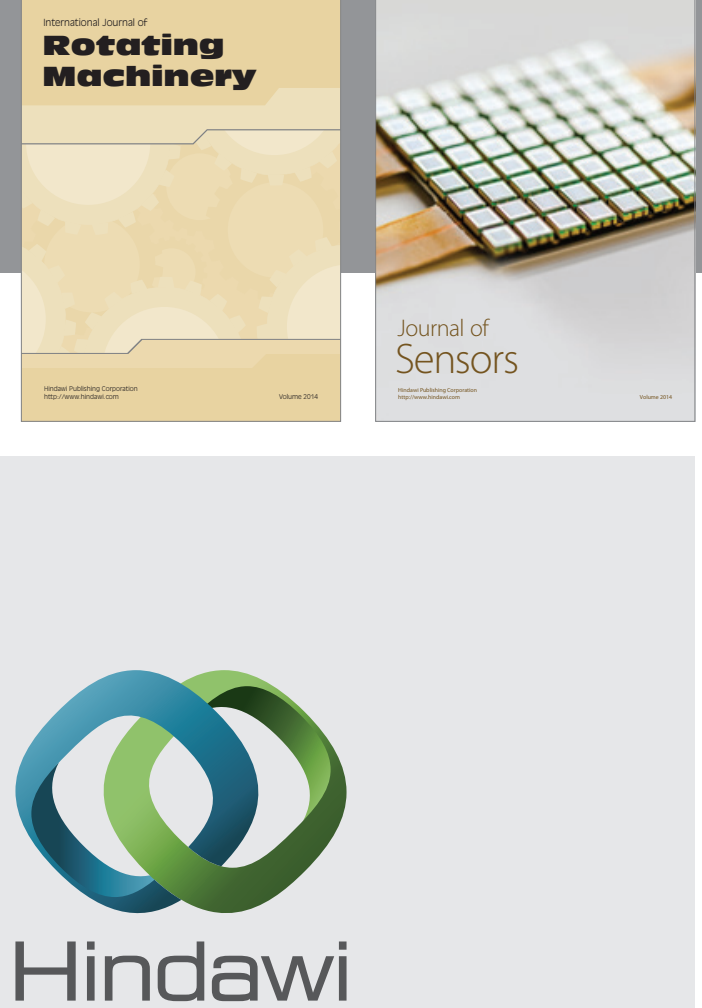

Submit your manuscripts at http://www.hindawi.com
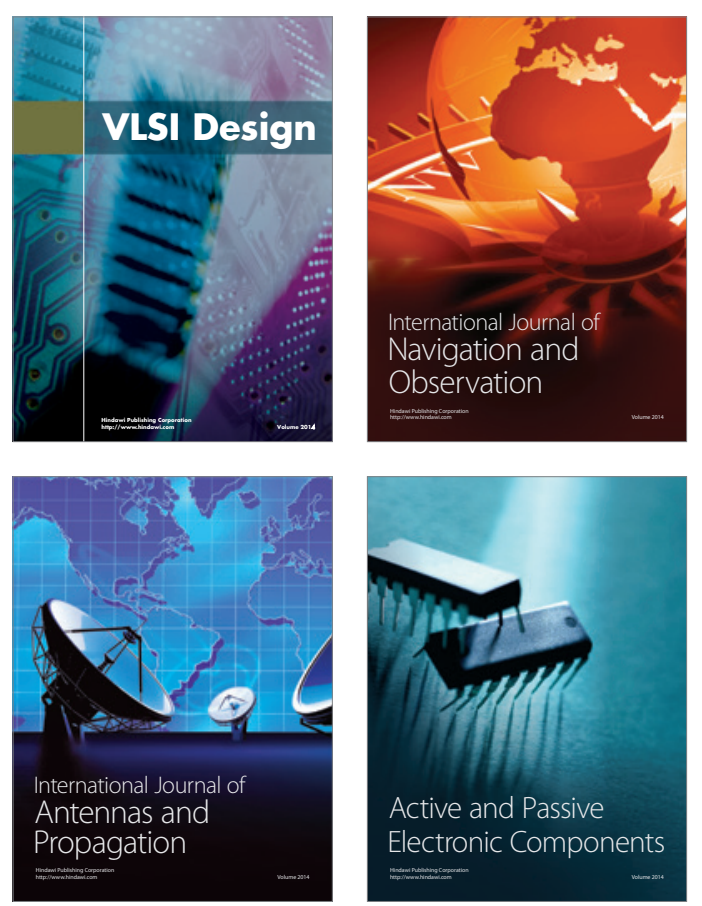
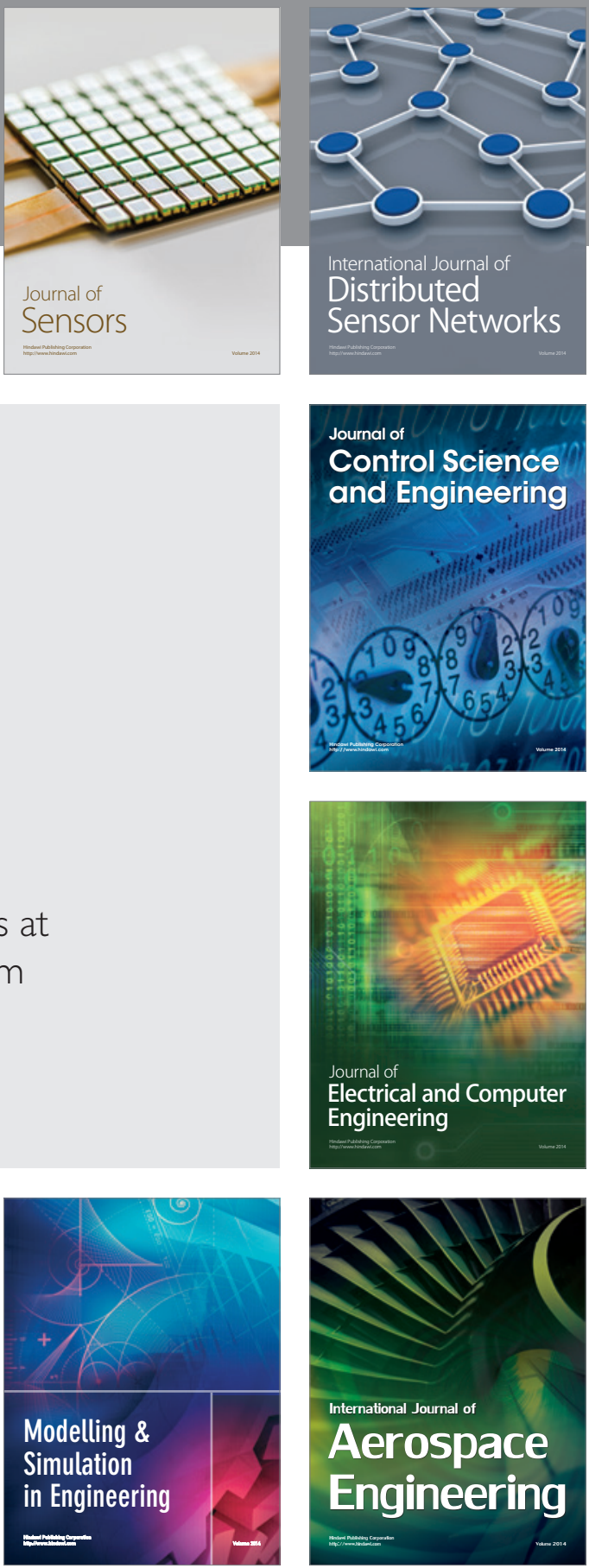

Journal of

Control Science

and Engineering
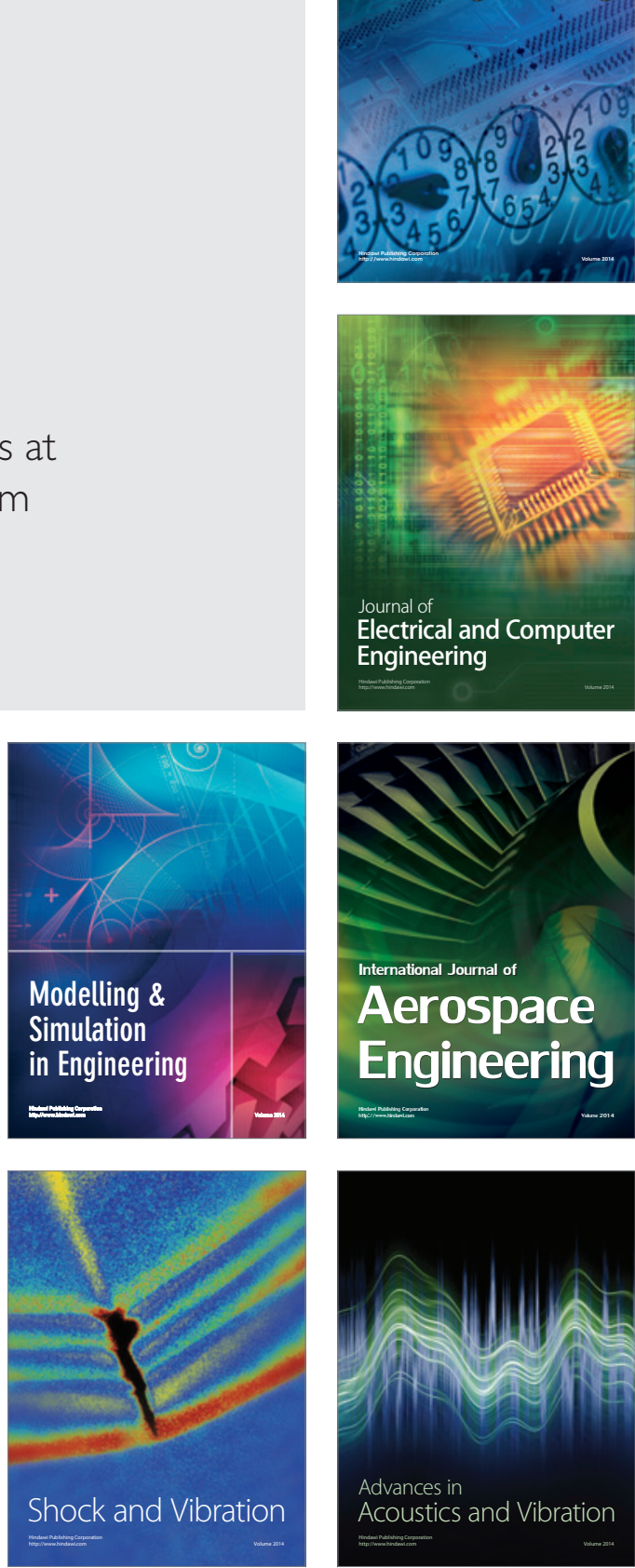